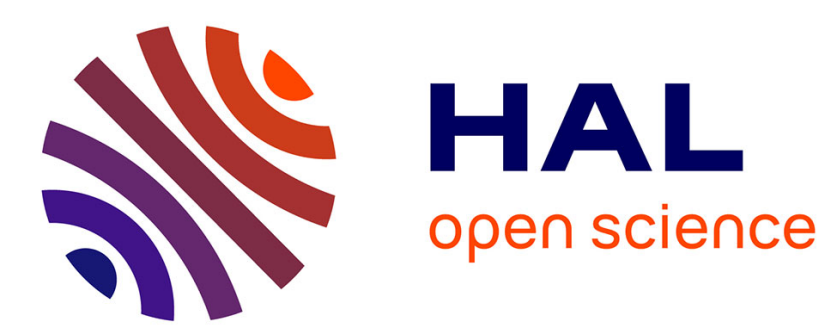

\title{
L'évolution de la tonnellerie en Côte-d'Or Jacques Berthod
}

\section{To cite this version:}

Jacques Berthod. L'évolution de la tonnellerie en Côte-d'Or. Revue forestière française, 1969, 21 (6), pp.585-587. 10.4267/2042/20302 . hal-03385939

\section{HAL Id: hal-03385939 \\ https://hal.science/hal-03385939}

Submitted on 19 Oct 2021

HAL is a multi-disciplinary open access archive for the deposit and dissemination of scientific research documents, whether they are published or not. The documents may come from teaching and research institutions in France or abroad, or from public or private research centers.
L'archive ouverte pluridisciplinaire HAL, est destinée au dépôt et à la diffusion de documents scientifiques de niveau recherche, publiés ou non, émanant des établissements d'enseignement et de recherche français ou étrangers, des laboratoires publics ou privés. 


\title{
LA TONNELLERIE EN BOURGOGNE
}

Class. Oxford $834.3(44 \times \mathrm{G1}-47)$

\author{
J. BERTHOD
}

Class. Oxford $834.3(44 \times$ G $1-47)$

La Bourgogne est par excellence le pays du vin et qui dit vin, dit en même temps tonnellerie, ou plus précisément, disait. Depuis le début du siécle en effet, cette prolession est entrée dans une période de déclin très net. Le nombre de tonneliers diminue d'année en année. Le fût traditionnel est concurrencé et souvent remplacé par des récipients en ciment ou en métal inoxydable dont les procédès de fabrication revêtent davantage un caractére industriel qu'artisanal et quı de ce fait s'avèrent extrêmement compétitifs quant aux prix. En outre, la fabrication des tonneaux ne peut ètre le fait d'une main-d'œuvre non spécialisée. Elle exige un personnel très qualifié. La qualité des produits est fonction pour une part importante de l'habileté de l'ouvrier. Or, cette main-d'œuvre se raréfie au fur et à mesure que la tonnellerie décline. Enfin, le tonnelier a de plus en plus de mal à supporter l'investissement important et néanmoins indispensable qu'est le stockage de la matière première.

Ce déclin se poursuivra-t-il inéluctablement? La profession ne sera-t-elle bientôt qu'un métier mort appartenant à un passé irrémédiablement révolu ? Ces questions, tous les tonneliers peuvent se les poser. En Côte d'or, cinq d'entre eux y ont répondu, d'une façon originale et dynamique, qui permet d'espérer un renouveau de la tonnellerie française.

Le 4 octobre 1968, naissait en effet le groupement d'intérêt économique "Les Tonnelleries de Bourgogne", groupement créé par cinq tonneliers de la Côte d'Or. Après concertation, les membres du groupe avaient décidé d'acheter en commun les merrains fendus, chez les producteurs du Limousin de préfèrence, et de coopérer chacun avec ses moyens propres pour exécuter les commandes importantes, ne se rèservant à titre individuel que la clientèle régionale. Enfin, fut établi un programme de répartition du travail, selon les commandes fermes ou probables.

\section{Genèse du groupement}

Fait curieux, c'est un diplomate américain qui est à l'origine de cette initiative. S'ètant aperçu que les plants cultivés en Californie et en Côte d'Or étaient de même qualité, ayant de plus remarqué que la nature du sol était semblable de part et d'autre, ce diplomate, propriétaire de vignobles outre-Atlantique, visita la Bourgogne et à cette occasion, étudia de très près les méthodes vinicoles qui y sont emplovées. Il acquit alors la conviction que, si le fût de bois était indispensable pour obtenir une bonne vinification, seul le chêne pouvait en garantir l'heureuse évolution. Passant trẻs vite de la conviction aux actes, il commanda des fûts à un tonnelier de Nuits-Saint-Georges.

Gette importation en Californie suscita, dans cet Etat, un très vif intérêt de la part des viticulteurs amèricains qui, eux aussi, constatèrent que leurs vins s'amélioraient s'ils étaient conservés et vieillis dans des fûts de chêne neuts, de préférence à toute autre sorte de récipient. Afin d'étayer cette constatation sur des bases scientifiques, des professeurs en œnologie furent envoyés en France et chargés de visiter les forêts renommées par leurs chênes pour sélectionner les variétés les plus à même d'assurer une excellente vinification.

D'après ces experts, le chéne rouvre et le chéne pédonculé que l'on rencontre dans le Limousin et les départements de la Nièvre et de l'Allier répondaient parfaitement grâce à leur texture serrẻe et à leur fibre résistante aux normes requises pour la fabrication d'une futaille de qualité. Par contre, en Côte d'Or, toujours selon eux, les chênes présenteraient pour cette fabrication un moindre intérêt. En particulier les chênes de la forêt de Citeaux oftriraient une texture trop lâche et leur fibre serait insuffisamment résistante, Quant à ceux issus de la région de FontaineFrançaise ils auraient une trop forte teneur en tanin, ce qui aurait pour effet de donner une saveur amère au vin. C'est pourquoi, tenant compte de ces conclusions, les tonneliers du groupement décidèrent d'acheter leurs merrains fendus de prétérence chez les producteurs du Limousin. 
Les conclusions positives des experts incitèrent donc les viticulteurs américains à falre appel à la tonnellerie française. C'est pour rèpondre dans les mellleures conditions aux larges perspectives ouvertes par ce débouché nouveau que fut alors créé le groupement des tonnelleries de Bourgogne.

\section{Activité du groupement}

Antérieurement à la constitution du groupement, la production annuelle de ces clnq fabricants, était de 2000 a 3000 pièces. Pour 1969, la production escomptée s'élévera a 5000 pièces, soit près du double. 2000 pièces seront exportées vers le marché américain. Mais il est bien évident que pour connattre un regain d'activité, la futaille française ne peut se satisfaire d'un seul débouché si important soit-il. Le groupement exporte donc vers d'autres pays. Cette année 700 fûts d'une capacité de 300 litres partiront pour l'U.R.S.S. aprés avoir été remplis d'alcool par des maisons de Cognac.

Les tonnelleries de Bourgogne semblent décidées á prospecter non seulement le marché des vins, mais aussi celui des alcools. En 1968 une maison écossaise n'avait pu se procurer en Bourgogne 10000 fôts à whisky. L'éventualité d'honorer des commandes de ce type fut alors envisagée et le groupement s'emploie depuis lors, à produire aussi des fûts capables de conserver les alcools. Ceux-ci doivent répondre aux exigences suivantes :

- capacité 300 litres (d'oủ la nécessitè de les fabriquer à partir de merrains de $1 \mathrm{~m} 10$ de long au lieu de $0 \mathrm{~m} \mathrm{90)}$.

- durée de séjour en futs : 6 ans au lieu de 2 en moyenne.

Enfln, les fûts françals commencent à étre recherchés par les producteurs de vins de quallté

M. SIRUGUE, tonnelier à Nults-Salnt-George, emballe dans du carton les fûts neufs quil seront expèdiès, vides, en Californie.

PHOTO GASTON DESGRANGES

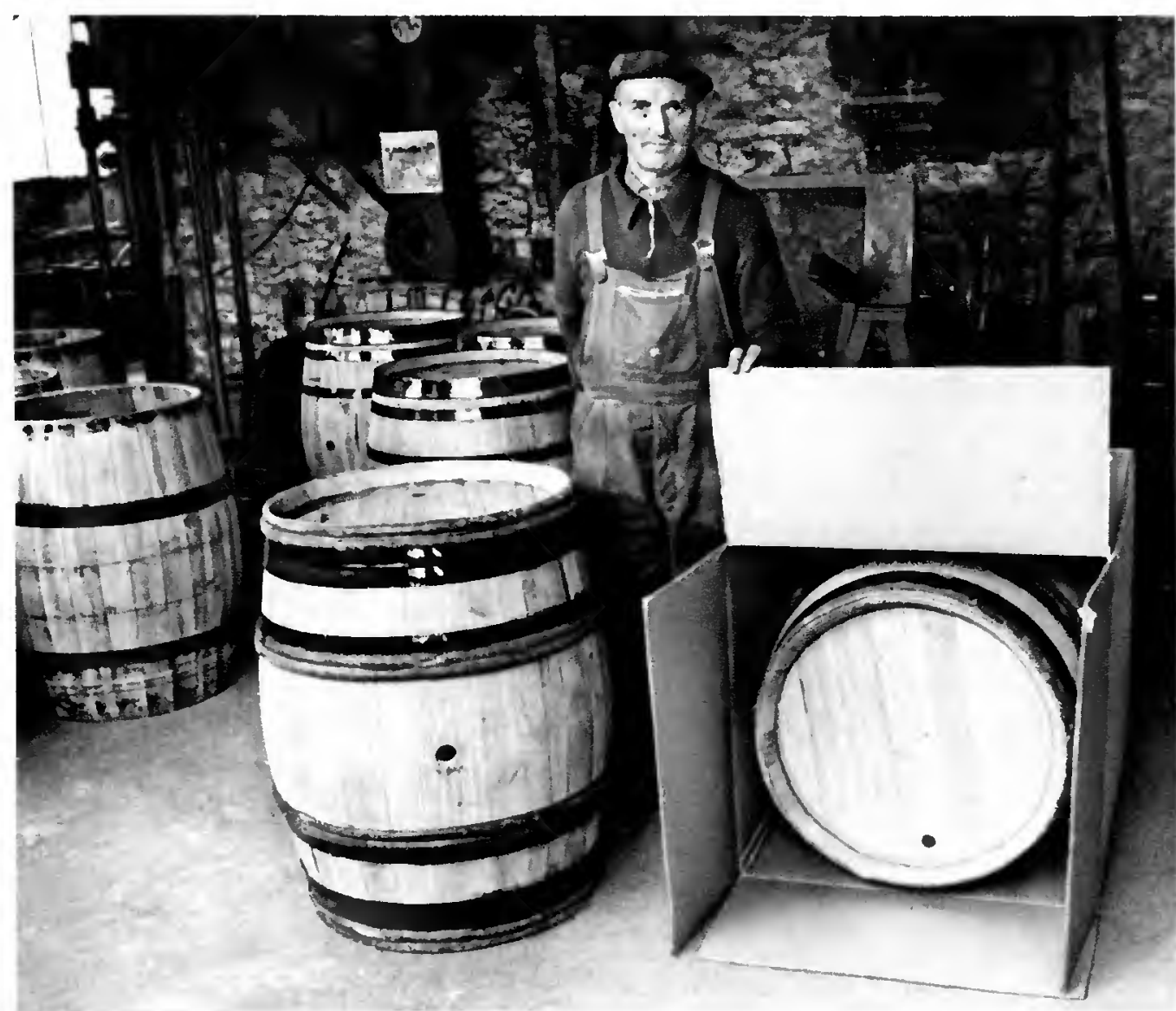


d'Australie, d'Argentine, d'Afrique du Sud et de Chypre. Cette liste n'est pas exhaustive et le groupement cherche activement à améliorer sa position sur l'ensemble des marchés, qui dans un proche avenir peuvent connaitre une importante expansion.

Les membres du groupe ont eu conscience que leur entreprise nécessitait le soutien de la publicité. Aussi dans de nombreux journaux, professionnels et autres, leur expérience a-t-elle étè décrite et commentèe en termes souvent élogieux.

\section{Autres tonneliers de la Côte d'Or}

Les tonnelleries de la Bourgogne ne se limitent pas pour autant à ce seul groupement; treize autres tonneliers pratiquent aussi cette profession, certains exportent des fûts démontés, fabriqués avec des merrains sciés, des frises ordinaires, le récipient est alors paraffiné. Un professionnel des environs de Beaune s'est même lancé dans la fabrication des bacs à sorbetières. Il exporte sa production en Chine.

Mais le tonnelier a de plus en plus de mal a faire face individuellement aux problèmes de rentabilité et d'expansion qui se posent à lui. Il semble que l'évolution actuelle qui conduisaît à la quasi extinction de la profession puisse étre freinée voire stoppée. A cet égard, l'exemple des tonnelleries de Bourgogne montre l'intérét que représente la formule du regroupement d'intérêt économique.

Les cinq professionnels qui l'ont créé ont réussi en un laps de temps trẻs court à doubler le chiftre de leur production. Par la pratique d'une politique d'achat commune et la répartition judicieuse de leur programme de travail, ils sont parvenus à créer une unité de production dynamique. La formule du groupement d'intérét économique semble présenter un grand intérêt pour la profession dans son ensemble. Elle est spécialement adaptée aux petites entreprises artisanales qui entendent conjuguer leurs activités. Elle permet une grande souplesse de fonctionnement et offre la possibilité d'évolutions ultérıeures vers la fusion, tout en permettant à ses membres de garder au départ, une certaine liberté d'action. Enfin, il est bien évident que sur le plan fiscal, des avantages substantiels peuvent être obtenus. La formule du groupement d'intérêt économique n'est pas magique et son application à la tonnellerie ne suffira sans doute pas à éliminer tous les problèmes qui se posent à la profession. Mais il n'en est pas moins évident que cette formule peut susciter a juste titre de très réels espoirs de renouveau.
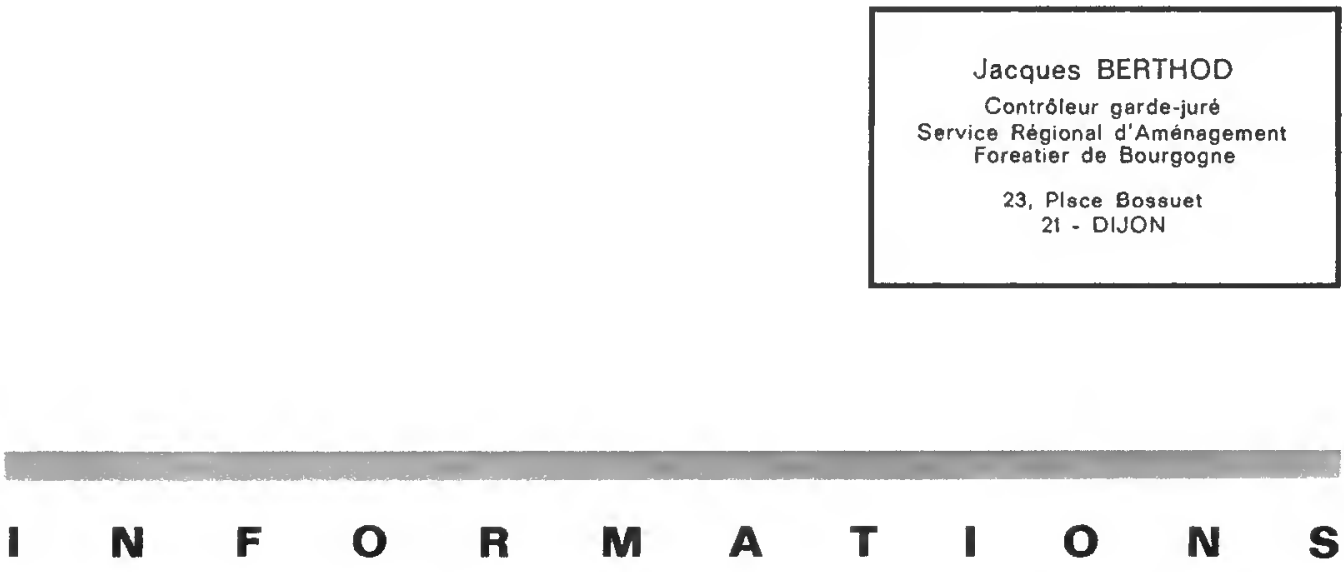

D'après une étude de la F.A.O. "Capacités mondiales de production de pâte et de papier 1969-1973 ", la capacité de production du papier et du carton dans le monde a augmenté de $5,6 \%$ par an depuis 1963 et pourrait augmenter d'environ $4,5 \%$ pour chacune des deux années à venir pour atteindre 145,6 millons de tonnes en 1971.

La capacité totale de production de pâte a augmenté de 5,6 \% par an depuis 1963 mais sa croissance ralentirait pour n'être plus que de $3,5 \%$ par an, ce qul la porterait à 122,3 millions de tonnes en 1971.

Ceci entraînerait une meilleure harmonie entre les deux capacités et un meilleur équilibre entre l'offre et la demande de pâte. 